\title{
Genesis Radiation Environment
}

\author{
Joseph I. Minow* \\ NASA Marshall Space Flight Center, Huntsville, AL 35812 USA \\ Richard L. Altstatt ${ }^{\dagger}$ and William C. Skipworth ${ }^{\ddagger}$ \\ Jacobs Engineering, Marshall Space Flight Center Group, Huntsville, AL 35812 USA
}

\begin{abstract}
The Genesis spacecraft launched on 8 August 2001 sampled solar wind environments at L1 from 2001 to 2004. After the Science Capsule door was opened, numerous foils and samples were exposed to the various solar wind environments during periods including slow solar wind from the streamer belts, fast solar wind flows from coronal holes, and coronal mass ejections. The Survey and Examination of Eroded Returned Surfaces (SEERS) program led by NASA's Space Environments and Effects program had initiated access for the space materials community to the remaining Science Capsule hardware after the science samples had been removed for evaluation of materials exposure to the space environment. This presentation will describe the process used to generate a reference radiation Genesis Radiation Environment developed for the SEERS program for use by the materials science community in their analyses of the Genesis hardware.
\end{abstract}

\section{Introduction}

$\mathrm{N}$ TASA's Space Environments and Effects (SEE) Program initiated the Surveying and Examination of Eroded Returned Surfaces (SEERS) Initiative in 2003. The goal of the Initiative was to provide leadership in the engineering analysis of returned flight hardware by supporting a comprehensive effort to understand environmental effects due to solar UV, ionizing radiation, plasmas, neutral contamination, meteoroids, and other conditions experienced during the mission (not primary science mission objectives). Initial efforts focused on coordinating the access by the materials engineering community to the remaining Genesis and Stardust hardware once the science materials were removed from the sample returned capsules. The Genesis spacecraft sampled solar wind environments at the Sun-Earth L1 point for a period of approximately three years before returning samples of solar wind materials in September 2004 [Wiens et al., 2002; Barraclough et al., 2004] and the Stardust spacecraft returned material from Comet P/Wild 2 and interstellar dust to Earth in January 2006. In support of the SEERS Initiative, the SEE Program funded development of a reference radiation environment for the Genesis spacecraft for use by materials engineers in their evaluation of the space exposed materials. This paper describes the status of this radiation environment development.

\section{Genesis Spacecraft and Plasma Data}

Genesis was launched on 8 August 2001 and inserted into a halo orbit about the Sun-Earth L1 point (Figure 1) on 16 November 2001 with sample collections obtained over an 884 day period from 3 December 2001 to 2 April 2004. Figure 2 shows the spacecraft with the solar arrays, the spacecraft bus with the Genesis Ion Monitor (GIM) and Electron Monitor (GEM) plasma instruments and support hardware, and the deployed Science Capsule in solar wind collection mode. The Science Capsule contains the solar wind collection materials and was designed to separate from the spacecraft bus after the science mission was complete

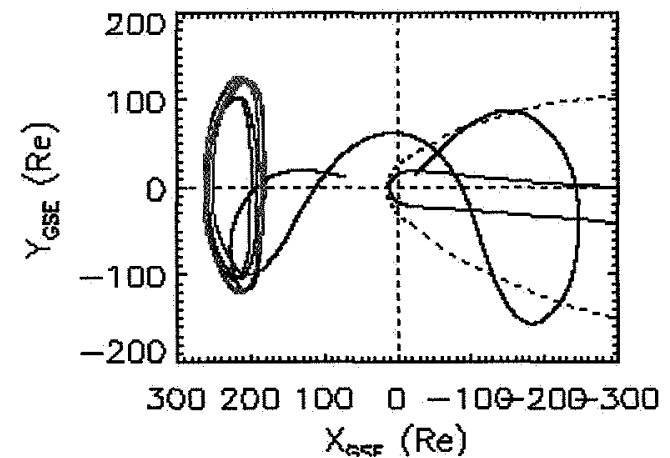

Figure 1. Genesis Orbit. Genesis trajectory from Earth to L1 (black) and for 30 November 2001 to 1 April 2004 including the period the Science Capsule was exposed to the solar wind (red).

\footnotetext{
*AST, Flight Vehicle Space Environments, Spacecraft and Vehicle Systems Department, NASA/MSFC/EV13, Huntsville, AL, Senior Member AIAA

${ }^{\dagger}$ Radiation Effects Engineer, Jacobs Engineering, MSFC Group, MSFC/EV13, Huntsville, AL

${ }^{\ddagger}$ Current position: Senior Software Engineer, Ameri-Tech International, Inc., Harvest, AL.
} 
and return to Earth.

The GEM and GIM plasma instruments [Barraclough et al., 2003; Neugebauer et al., 2003] were used to support the solar wind collection science by providing a real time monitor of solar wind conditions allowing the sample carriers to selectively collect material from coronal holes (fast $>500 \mathrm{~km} / \mathrm{sec}$ solar wind), interstreams (slow $<500 \mathrm{~km} / \mathrm{sec}$ solar wind), and coronal mass ejections. GIM is an electrostatic analyzer providing a complete set of ion flux measurements every 2.5 minutes over a $0.1 \mathrm{eV}$ to $14 \mathrm{keV}$ energy range and GEM is an electrostatic analyzer for measuring electron flux over the range of energies from 0.001 to $1.4 \mathrm{keV}$. Both instruments provided angular information on ion and electron flux but this data was not available for the radiation study. The radiation environments described here are based only on reconstructed environments derived from the GIM ion moments (Figure 3) provided by the PI and the public access Genesis data web site (http://genesis.lanl.gov/plots/index.html).

Ion moments derived from the GIM measurements are shown in Figure 3 from the start of instrument operations in August 2001 through the end of April 2004 during the period the Science Capsule was exposed to the solar wind highlighted in yellow. The ion density shown in the top panel of Figure 3 includes both the proton (black symbols) and helium (blue symbols) components of the ion flow which accounts for most of the solar wind mass. The helium density was computed from the $\mathrm{He}^{+} / \mathrm{H}^{+}$ratio reported in the Genesis GIM moments and the proton density. Gaps in the data set occur during periods when the GIM instrument was powered off for station keeping maneuvers and solar proton events. All the moment values were interpolated across these data gaps onto an evenly spaced set of time values (red)

The solar wind proton speed and temperatures are shown in the middle two panels. Corresponding helium parameters are not available in the moments so assumptions must be made to incorporate their effects into the reconstructed differential flux models. Observations of differential streaming velocities between solar wind protons and alpha particles have been reported with the alpha velocity exceeding the proton velocity by factors ranging from a few to $50 \mathrm{~km} / \mathrm{s}$ or more [Yermolaev and Stupin, 1997; Reisenfeld et al., 2001] with the largest differential streaming velocities occurring during periods of high speed solar wind flows and small differential alpha-proton velocity at $1 \mathrm{AU}$ for flows $<400$ to $500 \mathrm{~km} / \mathrm{s}$ [Neugebauer et al., 1996].

We have adopted a linear fit to alpha-proton differential streaming velocities (Figure 4) observed by instruments on the Helios [Marsch et al., 1982] and WIND [Steinberg et al., 1997] spacecraft to provide a method for deriving mean helium velocities from proton velocity moments available in the Genesis data set. The Marsh et al. [1982] values given in Figure 3 are the $0.95 \mathrm{AU}$ alpha-proton streaming values extracted from Neugebauer et al. [1996] and assigned to the midpoint of the proton velocity bins. Steinberg et al. [1997] in contrast reports average alpha-proton streaming values of $6.0 \pm 9.1 \mathrm{~km} / \mathrm{s}$ for $\mathrm{Vp}<400 \mathrm{~km} / \mathrm{s}, 20 \pm 17 \mathrm{~km} / \mathrm{s}$ for $400 \mathrm{~km} / \mathrm{s} \leq \mathrm{Vp} \leq 500 \mathrm{~km} / \mathrm{s}$, and $41 \pm 19 \mathrm{~km} / \mathrm{s}$ for $\mathrm{Vp}>500 \mathrm{~km} / \mathrm{s}$. In order to merge both data sets into the linear fit, we have computed the mean Genesis solar wind velocities $\mathrm{V}_{<400 \mathrm{~km} / \mathrm{s}}=349 \mathrm{~km} / \mathrm{s}$ and $\mathrm{V}_{>500 \mathrm{~km} / \mathrm{s}}=605 \mathrm{~km} / \mathrm{s}$ and assigned the corresponding Steinberg et al. [1997] alpha-proton differential streaming speeds of $\sim 6 \mathrm{~km} / \mathrm{s}$ and $\sim 41 \mathrm{~km} / \mathrm{s}$, respectively, to these values with the 400-500 $\mathrm{km} / \mathrm{s}$ differential streaming value assigned to a proton velocity of $450 \mathrm{~km} / \mathrm{s}$. The resulting linear fit for the differential velocity between alpha particles and protons is given by the equation

$$
\Delta V_{H e-P}=0.104 V_{P}-31.66 \mathrm{~km} / \mathrm{s}
$$

which is used in our model to provide the Genesis helium ion flow velocity. 


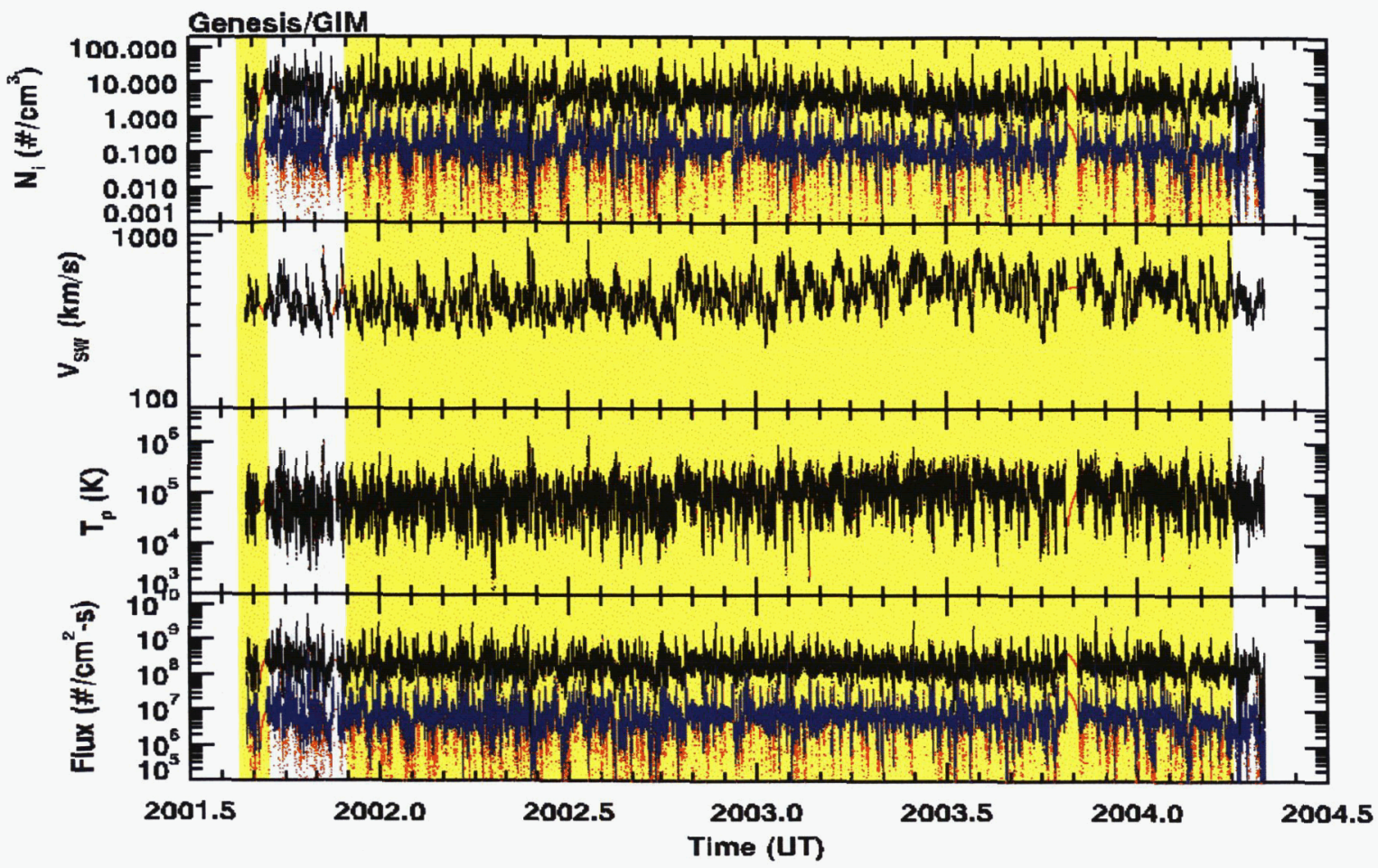

Figure 3. Genesis Ion Moments and Ion Flux. GIM ion moments (ion density $\mathrm{N}_{\mathrm{i}}$, solar wind ion velocity $\mathrm{V}_{\mathrm{SW}}$, and proton temperature $\mathrm{T}_{\mathrm{p}}$ ) are shown in the top three panels with the ion flux derived from the moments in the bottom panel. The period the Science Capsule was open is highlighted in yellow. Original GIM records are shown black (protons) and blue (helium) with interpolated values in red.

Helium to proton temperature ratios reported in the literature typically range in values from $\mathrm{T}_{\mathrm{He}} / \mathrm{T}_{\mathrm{H}} \sim 3$ to $\mathrm{T}_{\mathrm{He}} / \mathrm{T}_{\mathrm{H}} \sim 6$ depending on the nature of the solar wind flow regime. For example, Reisenfeld et al. [2001] use data from Feldman et al., [1977] to obtain an average ratio of $\mathrm{T}_{\mathrm{He}} / \mathrm{T}_{\mathrm{H}} \sim 4.9 \pm 1.8$ for all solar wind flows, $\mathrm{T}_{\mathrm{He}} / \mathrm{T}_{\mathrm{H}} \sim$ $6.2 \pm 1.3$ for flows $>650 \mathrm{~km} / \mathrm{s}$, and $\mathrm{T}_{\mathrm{He}} / \mathrm{T}_{\mathrm{H}} \sim 3.2 \pm 0.9$ for flows $<350 \mathrm{~km} / \mathrm{s}$. Marsch et al. [1982] report ratios of $\mathrm{T}_{\mathrm{He}} / \mathrm{T}_{\mathrm{H}} \sim 3.0$ for $>600 \mathrm{~km} / \mathrm{s}$ solar wind flows and $\mathrm{T}_{\mathrm{He}} / \mathrm{T}_{\mathrm{H}} \sim 2.3$ for solar wind flows $<400 \mathrm{~km} / \mathrm{s}$. Yermolaev and Stupin [1997] categorize solar wind data from the Prognoz -7 spacecraft into five solar wind flow regimes and report helium to proton temperature ratios of $\mathrm{T}_{\mathrm{He}} / \mathrm{T}_{\mathrm{H}} \sim 2.7 \pm 3.0$ for the heliospheric current sheet, $\mathrm{T}_{\mathrm{He}} / \mathrm{T}_{\mathrm{H}} \sim 4.2 \pm 3.6$ for coronal streamers, $\mathrm{T}_{\mathrm{He}} / \mathrm{T}_{\mathrm{H}} \sim 4.4 \pm 3.0$ for coronal holes, $\mathrm{T}_{\mathrm{He}} / \mathrm{T}_{\mathrm{H}} \sim 3.3 \pm 2.6$ for shocked solar wind, and $\mathrm{T}_{\mathrm{He}} / \mathrm{T}_{\mathrm{H}} \sim$ $3.9 \pm 2.8$ for coronal mass ejections. For the results presented in this paper, we will adopt the ratio $T_{\mathrm{He}} / \mathrm{T}_{\mathrm{H}} \sim 4.0$ independent of solar wind velocity.

Proton (black) and helium (blue) flux is presented in the bottom panel and is plotted over the interpolated flux values (red) that are based on the solar wind ram flux $\left(\sim \mathrm{V}_{\mathrm{Sw}} * \mathrm{~N}_{\mathrm{i}}\right)$. The flux exhibits nearly the same variability as the ion density due to the inverse relationship between solar wind velocity and density [Burlaga and Ogilvie, 1970] and the relatively smaller variation in solar wind velocity. For example, the mean and standard deviation of the proton density, flow velocity, and flux are $5.87 \pm 5.25 \mathrm{protons} / \mathrm{cm} 3,468 \pm 118 \mathrm{~km} / \mathrm{s}$, and $2.54 \pm 2.06 \times 10^{8}$ protons $/ \mathrm{cm} 2$ $\mathrm{s}$, respectively. The large flux variability is driven by the density contribution.

Collector materials were exposed to the solar wind from 17 August 2001 to 15 September 2001 and from 26 November 2001 to 1 April 2004 [Barraclough et al., 2004] for a total of approximately 886 days. Preliminary estimates of the total ion exposure during the mission report the interior of the Science Capsule accumulated a fluence of $1.90 \times 10^{16} \mathrm{H}^{+} / \mathrm{cm}^{2}, 9.80 \times 10^{14} \mathrm{He}^{++} / \mathrm{cm}^{2}$ with an error of $\pm 20 \%$ during the mission [Reisenfeld et al., 2006]. 


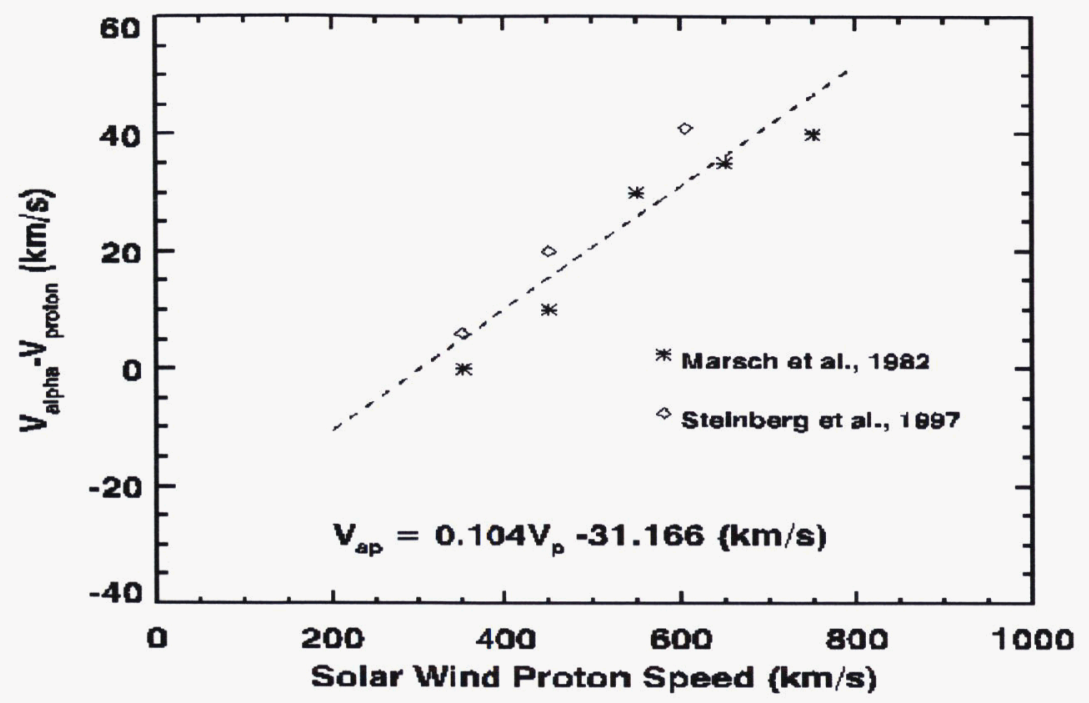

Figure 4. Differential Alpha-Proton Streaming Velocity. Linar model for the differential velocity $\mathrm{V}_{\text {ap }}$ between solar wind alpha particles and protons at $1 \mathrm{AU}$.

\section{Mean Surface Radiation Dose Estimate}

A simple order of magnitude analysis of the surface radiation dose generated by solar wind hydrogen and helium ions can be accomplished using the reconstructed environment described in the previous section. For this analyses, a period of record of approximately 980 days in length encompassing the complete set of GIM records shown in Figure 2 from DOY 236.68 in 2001 through DOY 122.00 in 2004 are used to characterize the ion flux environment. Mean values of the solar wind proton and helium density, velocity, number flux, and impact energy computed for the period are given in Table 1. Mean helium to hydrogen $\left\langle\mathrm{N}_{\mathrm{He}^{+}+}\right\rangle /\left\langle\mathrm{N}_{\mathrm{H}^{+}}\right\rangle$number density ratios are approximately 0.034 for this period consistent with values typically reported for solar wind flows. Helium flux and impact energies are greater than the corresponding hydrogen values by a factor greater than what would be expected if the ratios were due solely to the atomic mass ratio mass $\mathrm{He}^{++} / \mathrm{mass}_{\mathrm{He}++}=3.97$. The discrepancy is due to the approximately $18 \mathrm{~km} / \mathrm{s}$ mean alpha-proton differential streaming velocity and incorporating the effect of the streaming velocity into the analysis increases the mean helium flux to the $9.12 \times 10^{6} \mathrm{He}^{++} / \mathrm{cm}^{2}$-s value given in Table 1 from the smaller value of $8.84 \times 10^{6} \mathrm{He}^{+} / \mathrm{cm}^{2}-\mathrm{s}$ which would have resulted if the helium velocity was assumed to be the same as the proton velocity. In addition, the mean helium impact velocity would have been $4.87 \mathrm{keV}$ instead of $5.27 \mathrm{keV}$ as reported in Table 1 if the differential streaming velocity was not included in the model.

Dose (energy per unit mass) is computed from the mean particle flux values in Table 1 and knowledge of the depth penetration depth of the $\mathrm{H}^{+}, \mathrm{He}^{++}$ions into materials. TRIM calculations [Ziegler et al., 1985] for $1.22 \mathrm{keV} \mathrm{H}^{+}$ions and $5.27 \mathrm{keV} \mathrm{He}^{++}$ions on $1500 \AA$ thick aluminum and polyamide targets are shown in Figure 5 to estimate the range of solar wind ions in typical metals and polymers used in spacecraft construction. Simulation domains for each material are split in half with $\mathrm{H}^{+}$results shown in the top half and $\mathrm{He}^{++}$results in the bottom half of the simulation domain. The simulation used 10,000 particles for each species

\begin{tabular}{ccccc}
$\begin{array}{c}<\mathrm{N}> \\
\left(\# / \mathbf{c m}^{3}\right)\end{array}$ & $\begin{array}{c}<\mathrm{V}> \\
(\mathbf{k m} / \mathbf{s})\end{array}$ & $\begin{array}{c}<\text { flux } \\
\left(\# / \mathbf{c m}^{2}-\mathbf{s}\right)\end{array}$ & $\begin{array}{c}<\mathrm{E}> \\
(\mathbf{k e V})\end{array}$ \\
\hline $\mathrm{H}^{+}$ & 5.87 & 468 & $2.55 \times 10^{8}$ & 1.22 \\
$\mathrm{He}^{++}$ & 0.20 & 486 & $9.12 \times 10^{6}$ & 5.27
\end{tabular}

\begin{tabular}{ccc} 
Al & \multicolumn{2}{c}{$\rho=\mathbf{2 . 7 0 2} \mathbf{~ g} / \mathbf{c m}^{3}$} \\
$\Delta \mathbf{X H} \sim \mathbf{5 0 0} \AA$ & $\Delta \mathbf{X H e} \sim \mathbf{1 0 0 0} \AA$ \\
& $<$ dose rate> & <annual dose> \\
\hline $\mathrm{H}^{+}$ & $3.56 \mathrm{~Gy} / \mathrm{s}$ & $112.3 \mathrm{MGy} / \mathrm{yr}$ \\
$\mathrm{He}^{++}$ & $0.29 \mathrm{~Gy} / \mathrm{s}$ & $9.0 \mathrm{MGy} / \mathrm{yr}$
\end{tabular}

\begin{tabular}{|c|c|c|}
\hline \multicolumn{3}{|c|}{$\begin{array}{cl}\text { Polyimide } & \rho=1.430 \mathrm{~g} / \mathrm{cm}^{3} \\
\Delta \mathrm{XH} \sim 750 \AA & \Delta \mathrm{XHe} \sim 1250 \AA\end{array}$} \\
\hline & $<$ dose rate $>$ & $<$ annual dose $>$ \\
\hline $\mathrm{H}^{+}$ & $4.49 \mathrm{~Gy} / \mathrm{s}$ & $141.5 \mathrm{MGy} / \mathrm{yr}$ \\
\hline $\mathrm{He}^{++}$ & $0.54 \mathrm{~Gy} / \mathrm{s}$ & $17.0 \mathrm{MGy} / \mathrm{yr}$ \\
\hline
\end{tabular}



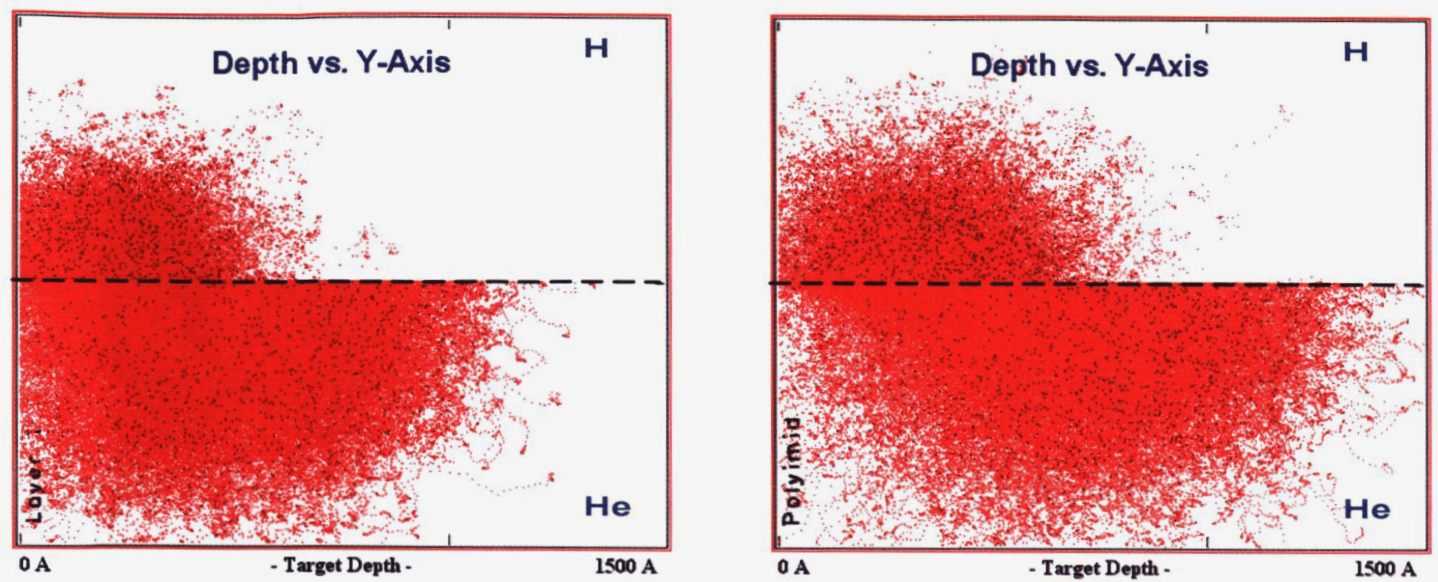

a) $\mathrm{H}$, He on Aluminum

b) H.He on Polyimide

Figure 5. TRIM Damage Analysis. TRIM Monte Carlo results showing penetration depth of $1.22 \mathrm{keV}$ $\mathrm{H}$ and $5.27 \mathrm{keV} \mathrm{He}$ in (a) aluminum and (b) polyamide $1500 \AA$ thick materials.

and the results shown in Figure 4 are the locations of ion collisions from the "ion distribution and quick calculation of damage" analysis option of the TRIM code. Penetration depths for the $1.22 \mathrm{keV} \mathrm{H}^{+}$and $5.27 \mathrm{keV} \mathrm{He}^{++}$ions are on the order of $500 \AA$ and $1000 \AA$, respectively, for aluminum and $750 \AA$ and $1250 \AA$, respectively, for polyamide. Dose for each species is determined from the ion flux, penetration depth, and material properties with the relationship

$$
\operatorname{Dose}(\text { Gray })=\frac{\Gamma E}{\rho \Delta x}
$$

where $\Gamma$ is the ion flux, $\mathrm{E}$ the mean ion impact energy, and $\rho$ the material density given in Table 1 and $\Delta \mathrm{x}$ is the ion penetration depth derived from Figure 5. This analysis assumes the ion energy is uniformly distributed over the penetration depth obtained from the TRIM analysis.

Mean dose rates and annual dose given in Table 1 are relatively large values but of course the potential material degradation only impacts the first $0.05 \mu \mathrm{m}$ to $0.15 \mu \mathrm{m}$ of the exposed materials surfaces. Optical properties including solar absorptivity $\left(\alpha_{\mathrm{S}}\right)$ and emissivity $(\varepsilon)$ coefficients are known to be sensitive to the surface condition of materials [c.f., Karam, 1998]. Evaluation of surface radiation dose is useful to determine potential impacts to materials used as thermal control coatings and structural elements to assure that optical properties remain within the required levels for the spacecraft to meet thermal requirements during the full mission lifetime. In the next section we describe a more sophisticated technique of deriving a reference radiation environment including the energy distribution of electrons, protons, and alpha partices which yields not only surface dose but can be used to compute dose as a function of depth in materials as well.

\section{Genesis Spectral Radiation Model}

A more sophisticated technique for producing a reference radiation environment for the Genesis spacecraft is to derive models for the differential flux spectra using plasma moments as inputs to Kappa velocity distribution functions [c.f, Chan et al., 1977; Garrett and DeForest, 1979; Kane et al., 1995; Minow et al., 2004, 2005] and constraining the high-energy, non-thermal tails with energetic flux measurements [Garrett and Hoffman, 2000; Collier et al., 1996; Maksimovic et al., 1997; Chotoo et al., 1998]. Plasma number density $\mathrm{N}_{\mathrm{i}}$, temperature $\mathrm{T}_{\mathrm{i}}$, and bulk velocity $\mathrm{V}_{\mathrm{C}, \mathrm{i}}$ moments for each species (electron, proton, or helium) are used to compute the Kappa distribution functions for the $i^{\text {th }}$ species $f_{k, i}$

$$
f_{\kappa, i}(\mathbf{v})=\frac{n_{i}}{\left(\sqrt{\pi} \theta_{\kappa, i}\right)^{3}} \frac{\Gamma\left(\kappa_{i}+1\right)}{\sqrt{\kappa_{i}{ }^{3}} \Gamma\left(\kappa_{i}-\frac{1}{2}\right)}\left[1+\frac{\left|\mathbf{v}-\mathbf{v}_{c}\right|^{2}}{\kappa_{i} \theta_{\kappa, i}^{2}}\right]^{-(\kappa+1)} \quad \theta_{\kappa, i}=\sqrt{\frac{(2 \kappa-3) k}{\kappa_{i} m_{i}}}
$$




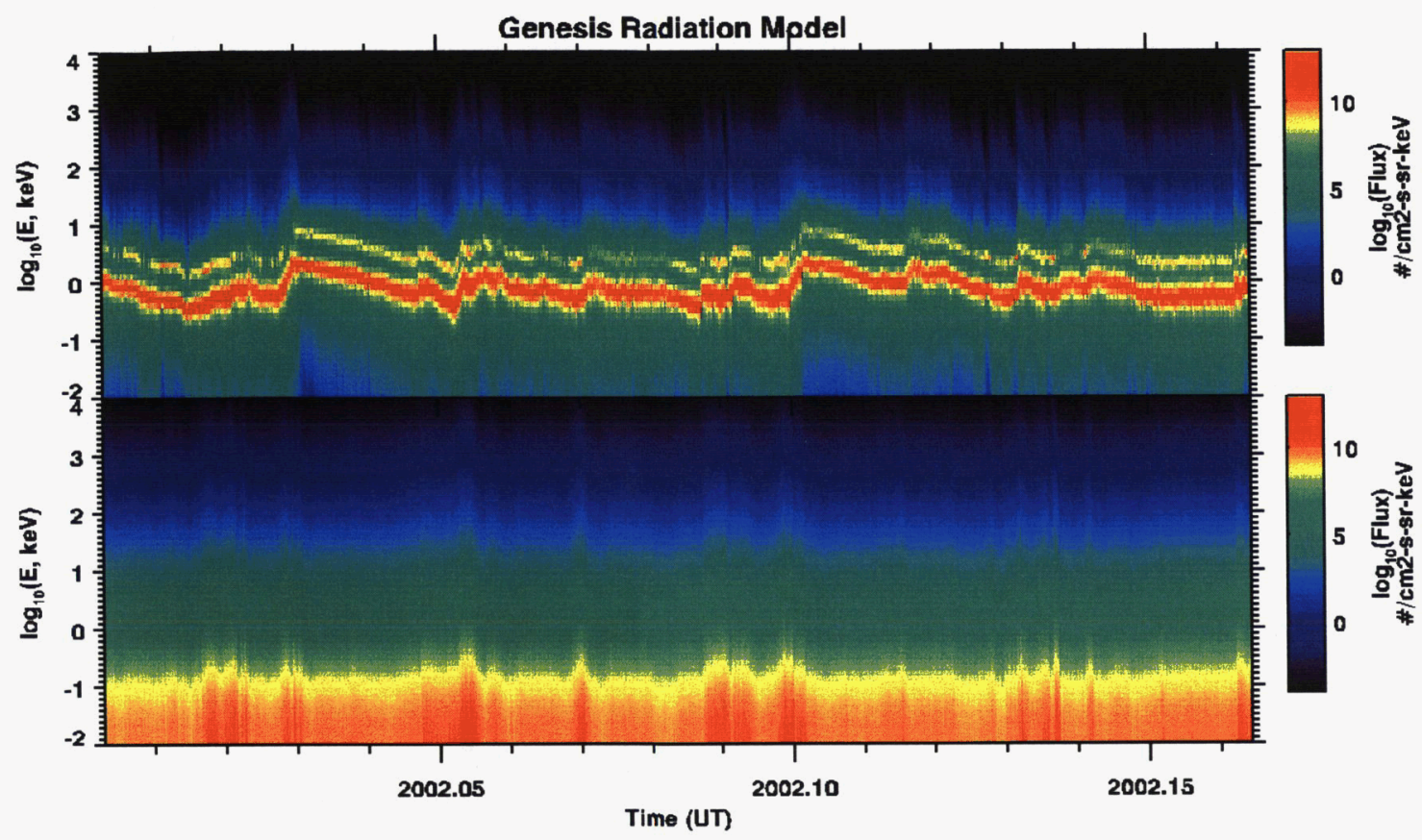

Figure 6. Reconstructed Genesis 2002 Environment. Ion (top) and electron (bottom) spectrograms for the reconstructed environment based on mean kappa values.

where $k_{B}$ is Boltzman's constant, $\Gamma(x)$ is the gamma function, $m_{i}$ the mass of species $i, \theta$ is the core thermal speed of the distributions [Collier et al., 1996], and $\kappa_{\mathrm{i}}$ is the Kappa parameter which must satisfy $\kappa_{\mathrm{i}}>3 / 2$ for all species. It is frequently advantageous to use bi-Maxwellian or even combinations of Maxwellian and Kappa distributions to reconstruct the differential flux from plasma moments. Differential flux $\mathrm{dJ} / \mathrm{dE}$ for species i (units of $\# / \mathrm{cm}^{2}-\mathrm{sec}-\mathrm{sr}-$ Joule) is derived from the distribution function $\mathrm{f}_{\mathrm{i}}$ (units of $\mathrm{As}^{3} / \mathrm{m}^{6}$ ) using the relationship

$$
\frac{d J_{i}}{d E}=\frac{\mathrm{v}^{2}}{\mathrm{~m}_{\mathrm{i}}} f_{i}(\mathrm{v})
$$

For the applications reported here, the non-thermal tails of the Kappa distributions which provide the internal charging environments are constrained by using differential flux measurements at energies from $100 \mathrm{keV}$ to 1000 $\mathrm{keV}$ assuring the environments are appropriate for internal charging analyses.

Figure 6 shows results from reconstructing differential flux spectra for environments impacting the sunward facing surface of the Genesis spacecraft during 2002. Solar wind ion environments are presented in a spectrogram format where the horizontal axis is time, the vertical axis is particle energy, and the differential flux is given by the color bar on the right. Genesis solar wind ion environments are sampled by the Genesis Ion Monitor instrument but ion flux data at energies greater than a few tens of $\mathrm{keV}$ required to constrain the $\kappa$ parameter for Kappa distribution function reconstructions are not available in the Genesis data set. A Kappa reconstruction is shown here where a mean value of $\kappa=3.5$ was assumed for all ion spectra based on mean $\kappa$ values reported in the scientific literature for solar wind helium ions which range from $\kappa=2.5$ to 5.8 [Chotoo et al., 1998; Collier, 1999]. 


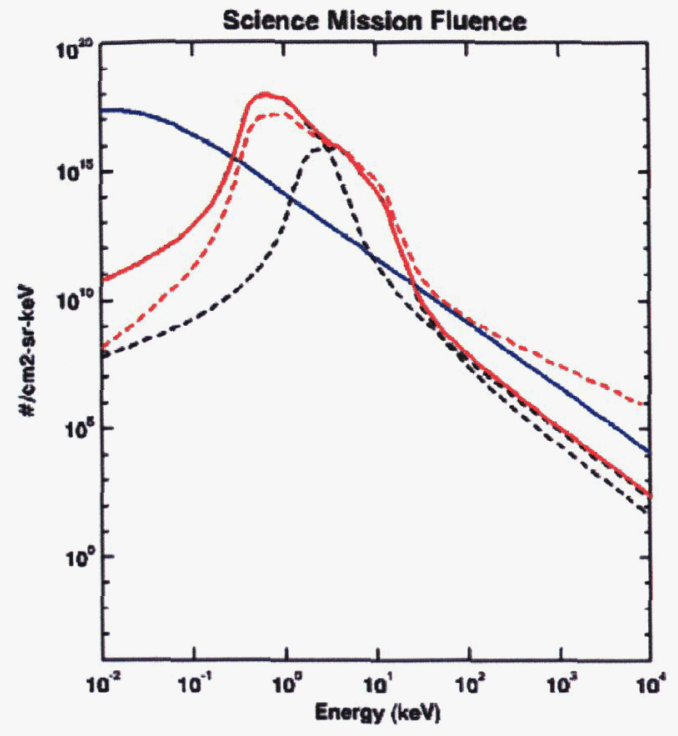

a)

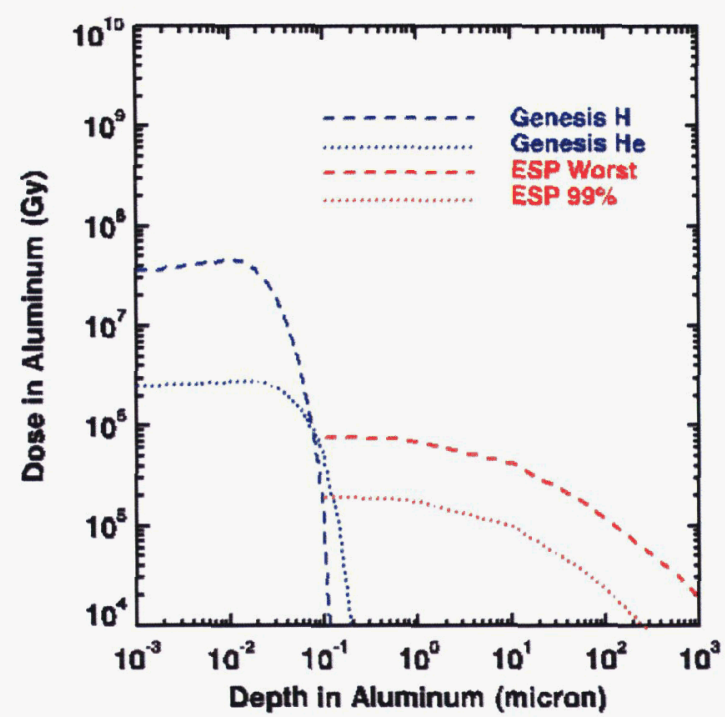

b)

Figure 7. Preliminary Genesis Mission Radiation Environment. (a) Fluence spectra obtained from integrating the modeled flux to the sun facing surface of the Genesis spacecraft. Ion fluence (solid red) exhibits a peak near $\sim 1$ to $\sim 10 \mathrm{keV}$ due to the proton (dashed red) and helium (dashed black) components while the electron (solid blue) fluence is nearly a power law over the energy range shown here. (b) Dose in aluminum as a function of depth in aluminum is shown for hydrogen and helium ions as well as electrons using the spectral model.

\section{Spectral Model Dose Analysis}

Integrating the differential flux provided by the reconstructed reference environment over the period of time the Science Capsule was exposed to the solar wind gives the electron and ion fluence spectra given in Figure 7-a. Ions (red) exhibit two peaks due to the effects of the hydrogen and helium ions while the electrons in this model exhibit a power law distribution over most of the energy range of interest.

Ion dose in aluminum as a function of depth in aluminum material derived from the spectral model is shown in figure 7-b. The fluence model from 6-a is transported through aluminum using the TRIM code and converting the ionization events into total ionizing dose. The near surface $(\leq 0.1 \mu \mathrm{m})$ dose values from the spectral model are less than the dose values given in the simple surface analysis described in Section III because the simple analysis assumes the entire ion flux for each ion species can be attributed to a single mono-energetic value. This is of course is not true because while the individual ion spectra exhibit sharp peaks at the $\mathrm{H}^{+}$and $\mathrm{He}^{+}$convection velocities, the peak in the ion fluence spectra are spread over an energy range of some 10's of kilovolts and the energy deposition in the material is correspondingly distributed. Use of the simple dose analysis will always overestimate the dose in the material but can be used as a bounding case when desired.

Radiation doses due to the solar wind ions are on the order a few $\sim 10^{6}$ Gy to thicknesses of a few tenths of a micron and drop rapidly after that due to the steep decrease in the solar wind spectrum. For comparison, the effect of energetic protons from solar energetic particle events are included in Figure 7-b. The Emission of Solar Proton (ESP) model [Xapsos et al., 1999,2000] is used to estimate contributions from extreme ( $99 \%$ confidence level) and ESP worst case solar proton events over the period 24 August 2001 to 1 April 2004, approximately the period the Science Capsule was open to the space environment. Dose in aluminum as function of depth in an infinite slab of aluminum is computed using the Shieldose-2 transport code [Seltzer, 1994]. Neither the worst case or the $99 \%$ confidence level ESP environments dominate the ion dose at the $0.1 \mu \mathrm{m}$ where the results can be directly compared. Energetic solar protons ( $>$ few MeV's) will pass through the thin surface layers of the materials depositing very little energy so it is not expected that the ESP model would provide additional dose at depths less than $0.1 \mu \mathrm{m}$ if the 
minimum energy of the model was extended below the current $1 \mathrm{MeV}$. Analysis of the electron environments is currently in progress and will be reported elsewhere.

\section{Summary}

We have described the development status of a reference radiation environment model for the space exposed material on the Genesis spacecraft's Science Capsule. The purpose of the work was originally to provide materials scientists with an accurate estimate of the radiation dose as a function of depth in the Genesis Science Capsule materials. In addition to this primary goal, the project also serves as a demonstration of a process to construct radiation environments for use in evaluating materials exposed to low energy $(<$ few $\mathrm{MeV}$ ) environments in interplanetary space.

\section{Acknowledgement}

Genesis Level 2 ion moments were provided courtesy of Roger Wiens and the Los Alamos Genesis Science Team. Financial support for this work was provided by NASA's Space Environments and Effects Program.

\section{References}

Barraclough B.L., Dors E.E., Abeyta R.A., Alexander J.F., Ameduri F.P., Baldonado J.R., Bame S.J., Casey P.J., Dirks G., Everett D.T., Gosling J.T., Grace K.M., Guerrero D.R., Kolar J.D., Kroesche J.L.Jr., Lockhart W.L., McComas D.J., Mietz D.E., Roese J., Sanders J., Steinberg J.T., Tokar R.L., Urdiales C., and Wiens R.C., The plasma ion and electron instruments for the Genesis Mission. Spa. Sci. Rev. 105, 627-660, 2003.

Barraclough B.L., Wiens R.C., Steinberg J.E., Reisenfeld D.B., Neugebauer M., Burnett D.S., Gosling J., and Bremmer R.R., The Genesis mission solar wind collection: solar-wind statistics over the period of collection, Lunar Planet Sci. XXXV, abstract 1763, 2004.

Burlaga, L.F., and K.W. Ogilvie, Magnetic and thermal pressure in the solar wind, Solar Phys., 15, 61, 1970.

Chan, K. W., K.W. Sawyer, J.I. Vette, A model of the near-earth plasma environment and application to the ISEE-A and -B orbit, NASA-TM-X-72611 (NSSDC/WDC-A-R/S-77-01, 19770701), July, 1977.

Chotoo, K., M.R. Collier, A.B. Galvin, D.C. Hamilton, and G. Gloeckler, Extended solar wind helium distribution functions in high-speed streams, J. Geophys. Res., 103, 17441 - 17445, 1998.

Collier, M.R., Evolution of kappa distributions under velocity space diffusion: A model for the observed relationship between their spectral parameters, J. Geophys. Res., 104, 28559-28564, 1999.

Feldman W.C., J.R. Asbridge, S.J. Bame, and J.T. Gosling, Plasma and Magnetic Fields from the Sun, in The Solar Output and its Variation, (ed.) Oran R. White, Colorado Associated University Press, Boulder, 1977.

Garrett, H.B., and A. Hoffman, Comparison of Spacecraft Charging Environments at the Earth, Jupiter, and Saturn, in 6th Spacecraft Charging Technology Conference, AFRL-VS-TR-20001578, 1 September 2000.

Karam, R.D., Satellite Thermal Control for Systems Engineers, Volume 181 Progress in Astronautics and Aeronautics, P. Zarchan (Ed.), American Institute of Aeronautics and Astronautics, Inc., Reston, VA, 1998.

Maksimovic, M., V. Pierrard, and P. Riley, Ulysses electron distributions fitted with Kappa functions, Geophys. Res. Lett., 24, 1151-1154, 1997.

Marsch, E., K.-H. Muhlhauser, H. Rosenbauer, R. Schwenn, and F.M. Neubauer, Solar wind helium ions: Observations of the Helios solar probes between 0.3 and 1 AU, J. Geophys. Res., 87, 35, 1982.

Minow, J.I., W.C.Blackwell,Jr., and A. Diekmann, Plasma Environment and Models for L2, ALAA Paper 20041079, 42nd AIAA Aerospace Sciences Meeting and Exhibit, Reno, NV, 5-8 January 2004.

Minow, J.I., L.N. Parker, and R.L. Altstatt, Radiation and internal charging environments for thin dielectrics in interplanetary space, presented at the $9^{\text {th }}$ Spacecraft Charging Technology Conference, Tsukuba, Japan, 4-8 April 2005.

Neugebauer, M., B.E. Goldstein, E.J. Smith, and W.C. Feldman, Ulysses observations of differential alpha-proton streaming in the solar wind, J. Geophys. Res., 101, 17047, 1996.

Neugebauer, M., Steinberg J.T., Tokar R.L., Barraclough B.L., Dors E.E., Wiens R.C., Gingerich D.E., Luckey D., and Whiteaker D.B., Genesis on-board determination of the solar wind flow regime, Spa. Sci. Rev. 105, 661679, 2003.

Reisenfeld, D.B., S.P. Gary, J.T. Gosling, J.T. Steinberg, D.J. McComas, B.E. Goldstein, and M. Neugebauer, Helium energetics in the high-latitude solar wind: Ulysses. observations, J. Geophys. Res., 106, 5693 - 5708, 2001.

Reisenfeld, D.B., R.C. Wiens, B.L. Barraclough, J.T. Steinberg, C. DeKoning, J. Raines, T. H. Zurbuchen, D.S. Burnett, The Genesis mission, the effects of solar wind conditions on the deposition and interpretation of the Genesis samples, Lunar Planet Sci., XXXVII, abstract 1830, 2006. 
Seltzer, S. M., Updated calculations for routine space-shielding radiation dose estimates: SHIELDOSE-2, NIST Publication NISTIR 5477, Gaithersburg, MD., December 1994.

Steinberg, J.T., A.J. Lazarus, K.W. Ogilvie, R. Lepping, and J. Byrnes, Differential flow between solar wind protons and alpha particles: First WIND observations, Geophys. Res. Lett., 23, $1183-1186,1996$.

Wiens R.C., Burnett D.S., Neugebauer M., Sasaki C., Sevilla D., Stansbery E., Clark B., Smith N., Oldham L., Barraclough B., Dors E.E., Steinberg J., Reisenfeld D.B., Nordholt J.E., Jurewicz A., and Cyr K., The Genesis solar wind sample return mission. EOS, Transactions Am. Geophys. Union 83, 229-234, 2002.

Xapsos, M. A., G. P. Summers, J. L. Barth, E. G. Stassinopoulos, and E. A. Burke, Probability Model for Worst Case Solar Proton Event Fluences, IEEE Trans. Nucl. Sci., 46, 1481-1485, 1999.

Xapsos, M. A., G. P. Summers, J. L. Barth, E. G. Stassinopoulos, and E. A. Burke, Probability Model for Cumulative Solar Proton Event Fluences, IEEE Trans. Nucl. Sci., 47, 486-490, 2000.

Yermolaev, Y.I., and V.V. Stupin, Helium abundance and dynamics in different types of solar wind streams: The Prognoz 7 observations, J. Geophys. Res., 102, 2125 - 2136, 1997.

Ziegler, J.F., J. P. Biersack and U. Littmark, The Stopping and Range of Ions in Solids, Pergamon Press, New York, 1985. 